\title{
Removing frequency dependent aberrations in acoustic microscopy
}

\section{Tommiska, Oskari Mikael}

IEEE

2019

Tommiska , O M , Meriläinen , A , Mäkinen , J M K , Hyvönen , J T J , Salmi , A \& Haeggström , E 2019 , Removing frequency dependent aberrations in acoustic microscopy . in 2019 IEEE International Ultrasonics Symposium (IUS). IEEE International Ultrasonics Symposium , IEEE , pp. 593-595 , IEEE International Ultrasonics Symposium , Glasgow , United Kingdom , 06/10/2019 . https://doi.org/10.1109/ULTSYM.2019.8925779

http://hdl.handle.net/10138/326121

https://doi.org/10.1109/ULTSYM.2019.8925779

acceptedVersion

Downloaded from Helda, University of Helsinki institutional repository.

This is an electronic reprint of the original article.

This reprint may differ from the original in pagination and typographic detail.

Please cite the original version. 


\section{Removing frequency dependent aberrations in acoustic microscopy}

\author{
Oskari Tommiska \\ Electronics lab., Physics dept. \\ Univ. of Helsinki \\ Helsinki, Finland
}

\author{
Antti Meriläinen \\ Electronics lab., Physics dept. \\ Univ. of Helsinki \\ Helsinki, Finland \\ Ari Salmi
Electronics lab., Physics dept.
Univ. of Helsinki \\ Helsinki, Finland
}

\author{
Joni Mäkinen \\ Electronics lab., Physics dept. \\ Univ. of Helsinki \\ Helsinki, Finland
}

\author{
Jere Hyvönen \\ Electronics lab., Physics dept. \\ Univ. of Helsinki \\ Helsinki, Finland
}

Edward Hæggström

Electronics lab., Physics dept.

Univ. of Helsinki

Helsinki, Finland

\begin{abstract}
In acoustic microscopy, a focused high frequency ultrasonic pulse is used to nondestructively image samples. The ultrasound signal is focused with an acoustic lens. In case of a broadband signal, the final image can feature aberrations, caused by frequency dependent changes in focal distance of the acoustic lens. By understanding the frequency dependency of the focal distance, one can remove such aberrations, resulting in higher image quality. We present results of our work towards removing the frequency dependent aberrations from an acoustic image.
\end{abstract}

Keywords-acoustic microscopy, acoustic imaging, frequency dependency, focal distance, aberrations

\section{INTRODUCTION}

Scanning acoustic microscopy (SAM) uses focused high frequency ultrasound to image samples on micrometer scale [1]. SAM determines both topology and material properties of a sample. Due to its nondestructive nature, acoustic microscopy is useful for imaging biological samples [2].

The imaging is done by measuring a focused ultrasound signal reflected back form the sample. An image is constructed by performing a $2 \mathrm{D}$-scan across the desired area. To obtain a high-quality image, it is critical to determine the focal length of the acoustic lens in use. Fig. 1 shows focusing of acoustic waves in immersion.

We previously studied the frequency dependency of the focal length with simulations and experimentally, and both approaches indicated that the focusing of the acoustic lens is frequency dependent [3].

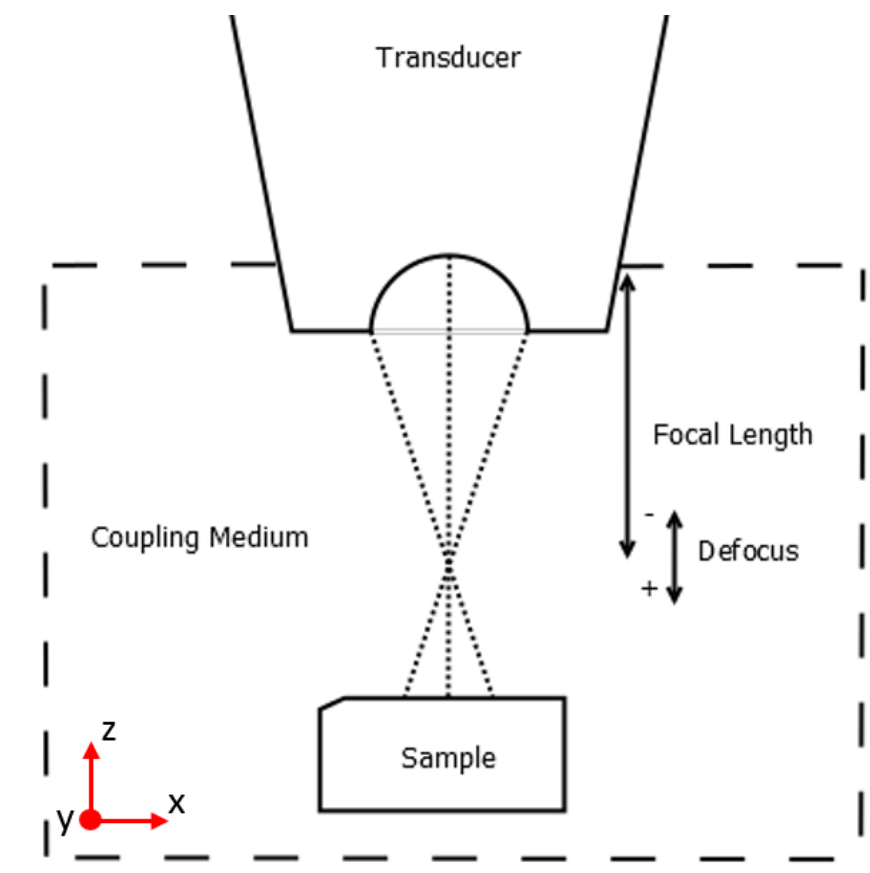

Fig. 1. Focusing of acoustic waves in immersion.

When measuring with a broadband signal, aberrations caused by a frequency-dependent focal length need to be addressed. In our method we do a 3D-scan of a sample and during postprocessing we enhance the image, by focusing each frequency component separately.

\section{METHODS}

Our SAM is described in [4]. An acoustic lens with $250 \mathrm{MHz}$ central frequency and numerical aperture of 0.5 was used.

To study the frequency dependency of the focal distance, a sample was scanned at different lens-sample distances. Our sample was the USAF 1951 microscope resolution test chart. The scan was $100 \times 300 \times 150 \mu \mathrm{m}^{3}$, with $1 \mu \mathrm{m}$ step size. The 
ultrasound signal was a broadband chirp with frequency band of 130-370 MHz.

The measured data was analyzed in MATLAB R2019a. To analyze the contribution of separate frequency components, the data was transformed into the frequency domain using fast Fourier transform. The transformed data was divided into narrow frequency components, each of which was analyzed separately.

The focal distance of each frequency component was solved at each scanned point by determining the distance, at which the reflected signal exhibited the highest amplitude. The accurate focal distance and the amplitude value were determined by fitting a gaussian curve near the peak value (Fig. 2).

This process was repeated for each frequency component. Fig. 3 shows an example of resulting peak amplitude versus distance for all frequency components within the transducer bandwidth. The pixel value was the sum of amplitude values across the frequency band.

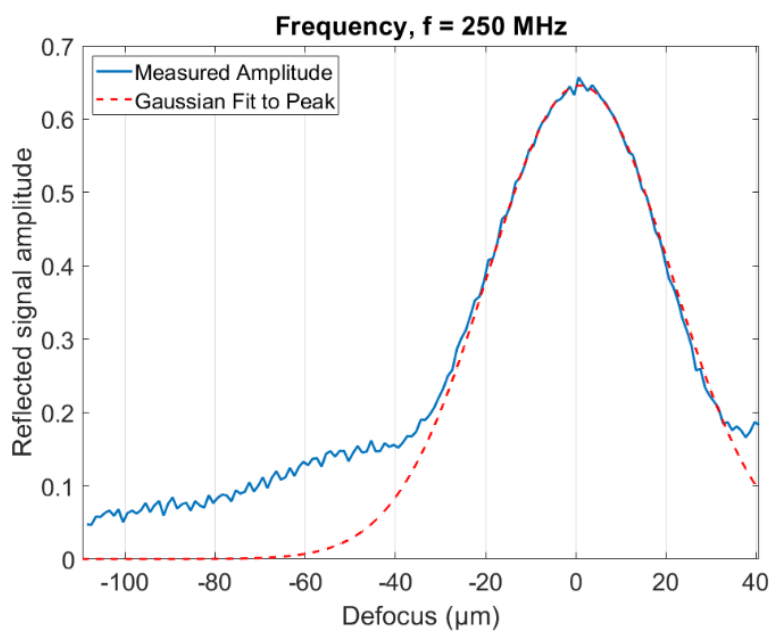

Fig. 2. Gaussian curve fit around the peak to determine the defocus of one frequency component at one location. The measured blue curve is for the central frequency $\mathrm{f}=250 \mathrm{MHz}$ at location $\mathrm{x}=1, \mathrm{y}=1$.

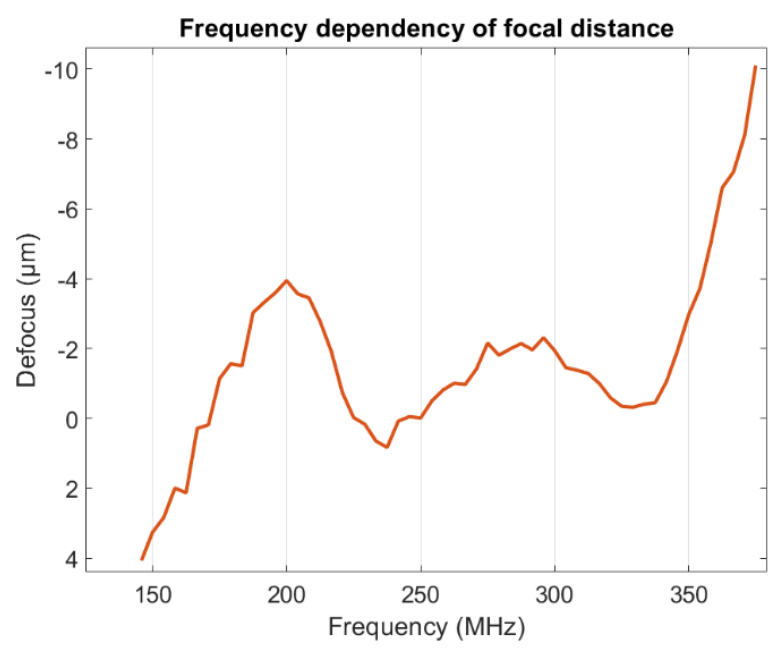

Fig. 3. Defocus of all analyzed frequency components at point $x=1, y=1$.
To verify any possible improvement in image quality, another image was produced, using the same experimental data. The produced reference image was created without considering the effect caused by the frequency dependency of the focal distance.

The reference image was constructed by first determining the focal distance without considering the frequency aberrations and then by averaging over images along the z-axis (Fig. 1). To make a fair comparison between two different methods, the number of averaged images was determined by calculating the contrast-to-noise ratio (CNR) and by comparing this CNR value to the corresponding $\mathrm{CNR}$ value of enhanced image.

\section{RESULTS AND DISCUSSION}

The enhanced image was compared with the reference image. A comparison between two images is presented in Fig. 4. The CNR value of each image is displayed. Qualitative visual comparison of the images suggests that the proposed method produces sharper images (Fig. 5).

Images were compared in detail by examining the surface profiles across the lines of the resolution test chart. Fig. 6 shows an example of a surface profile.
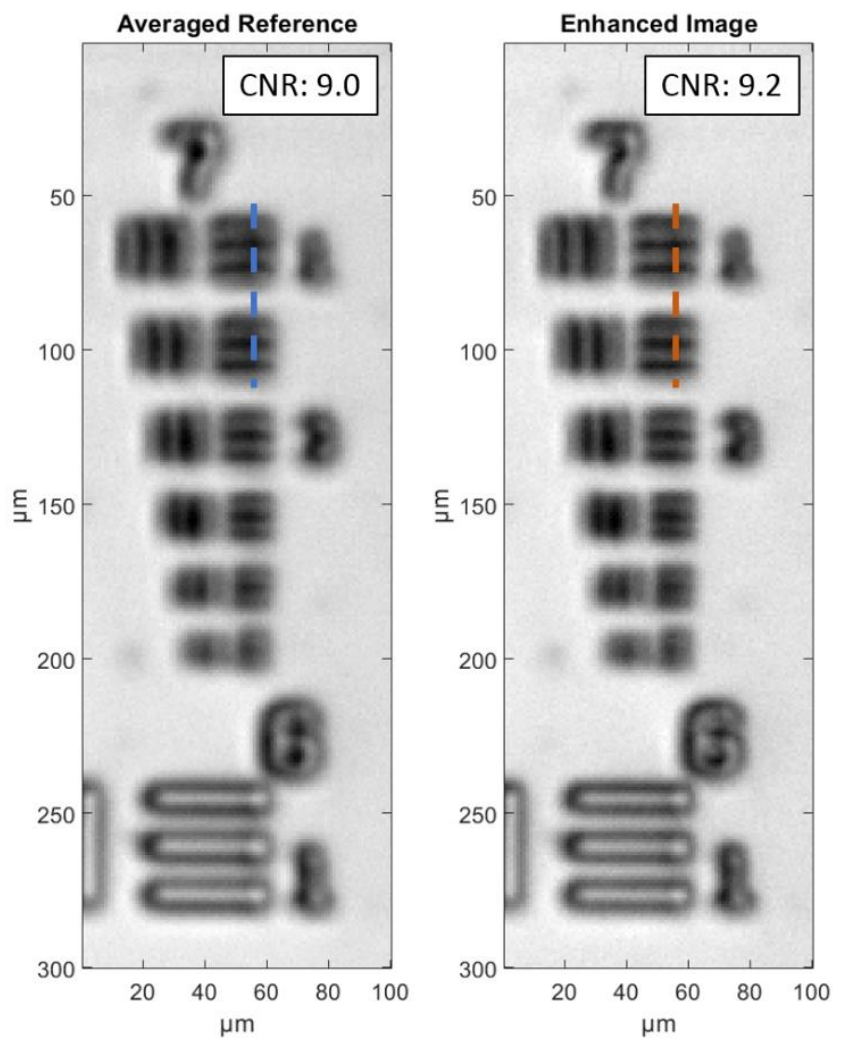

Fig. 4. Comparison of images: (left) image produced without considering the aberration, (right) image produced using the proposed method presented. Both the reference image and the enhanced image were constructed using 15 measured layers. Dashed lines show locations of surface profiles in Fig. 6. 


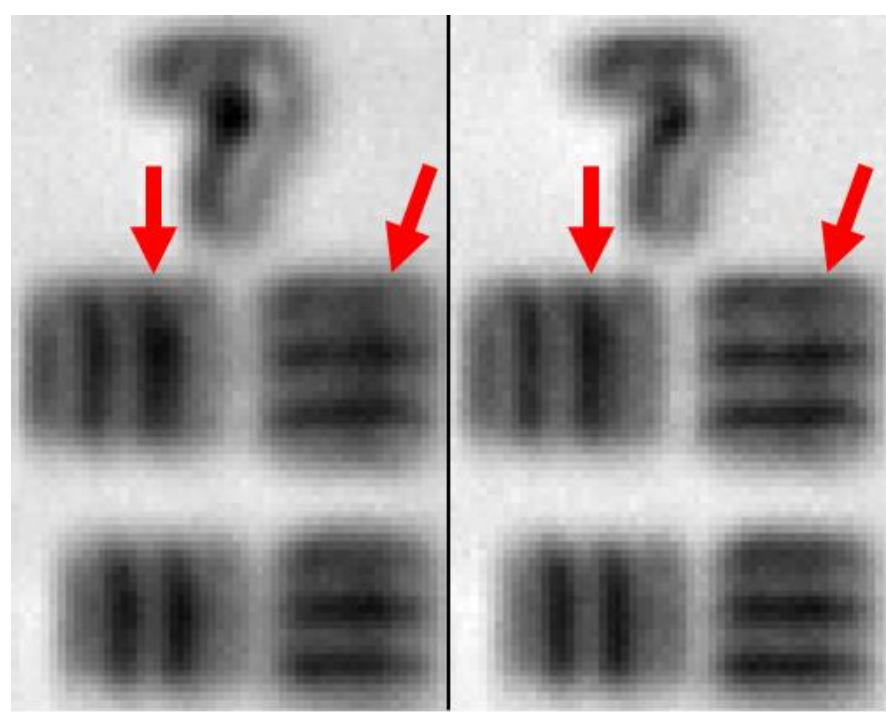

Fig. 5. Zoomed in detail: (left) reference image and (right) enhanced image. Comparing lines reveals the difference in sharpness.

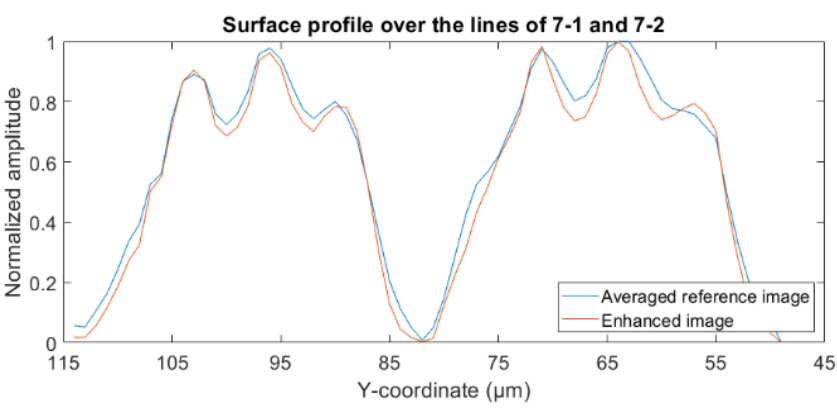

Fig. 6. Comparison of surface profiles of images in Fig. 4. Surface profiles taken along same line, across the lines of the goups 7-1 and 7-2 on resolution test chart.

The comparison confirms that the proposed method keeps the details sharper, when compared to the normal method with averaging. Near $y=55 \mu \mathrm{m}$ in Fig. 6, the frequency aberration method restores a detail, that would otherwise have been lost during traditional averaging.

Further comparisons were made between the enhanced image and images produced using the normal method. In these experiments a different number of images was employed during averaging. The enhanced image was also compared with the non-averaged reference image.

The noise in the reference images was greater than in the enhanced image in all cases where the number of averaged images was less than the number of averaged images in the reference image in Fig. 4. This was determined both by visually comparing figures and by calculating the CNR values. Furthermore, the blur was higher, thus reducing the sharpness of reference image in all cases with more averaging than in the reference image in Fig. 4.

\section{CONCLUSION}

The frequency aberration-based image enhancement method reduces noise without blurring the final image. The proposed method can improve the quality of acoustic images in cases where high resolution and low noise are important.

\section{REFERENCES}

[1] R. N. Johnston, A. Atalar, J. Heiserman, V. Jipson, C. F. Quate, Acoustic Microscopy: Resolution of Subcellular Detail, Proc. Natl. Acad. Sci. U.S.A., vol. 76(7), 1979

[2] R. A. Lemons, C. F. Quate, Acoustic Microscopy: Biomedical Applications, Science, vol. 188, 1975

[3] O. Tommiska, A. Meriläinen, J. Mäkinen, J. Hyvönen, A. Salmi, E. Hæggström, "Frequency dependencey of focal distance of scanning acoustic microscope lens", Physics Days, The 53rd annual meeting of the Finnish Physical Society, Helsinki, 2019

[4] A. I. Meriläinen, V. Kananen, C. Fridlund, J. Eskelinen, E. Hæggström, GHz Ultrasonics with Arbitrary Code Excitation, IEEE IUS 2013 